\title{
Avaliação multidimensional do usuário de drogas e a Escala de Gravidade de Dependência
}

\author{
Multidimensional evaluation of drug users and the Addiction Severity Index \\ Felix Kessler ${ }^{1}$, Sibele Faller ${ }^{2}$, Maria Lucia O. Souza-Formigoni ${ }^{3}$, Marcelo Santos Cruz ${ }^{4}$, Sílvia Brasiliano \\ Anderson Ravy Stolf ${ }^{6}$, Flavio Pechansky ${ }^{7}$
}

${ }^{1}$ Mestre. Vice-diretor, Centro de Pesquisa em Álcool e Drogas (CPAD), Universidade Federal do Rio Grande do Sul (UFRGS), Porto Alegre, RS. ${ }^{2}$ Psicóloga, CPAD, UFRGS. Mestre, UFRGS. ${ }^{3}$ Livre-docente. Professora, Departamento de Psicobiologia, Universidade Federal de São Paulo (UNIFESP), São Paulo, SP. ${ }^{4}$ Doutor. Psiquiatra, Programa de Estudos e Assistência ao Uso Indevido de Drogas (PROJAD), Instituto de Psiquiatria, Universidade Federal do Rio de Janeiro (UFRJ), Rio de Janeiro, RJ. ${ }^{5}$ Doutora. Psicóloga, Programa de Atenção à Mulher Dependente Química (PROMUD), Instituto de Psiquiatria, Faculdade de Medicina, Universidade de São Paulo (USP), São Paulo, SP. ${ }^{6}$ Psiquiatra. Assistente de pesquisa, CPAD, UFRGS. ${ }^{7}$ Doutor. Psiquiatra. Diretor, CPAD, UFRGS, e professor, UFRGS.

Este estudo foi desenvolvido no Centro de Pesquisa em Álcool e Drogas (CPAD), Universidade Federal do Rio Grande do Sul (UFRGS), Porto Alegre, RS.

Suporte financeiro: Secretaria Nacional de Políticas sobre Drogas (SENAD).

\section{Resumo}

Introdução: Antes de iniciar um tratamento para abuso de substâncias psicoativas (SPA), é primordial avaliar os prejuízos que estas acarretam na vida dos usuários. Considerando a complexidade dessa avaliação, o instrumento atualmente mais utilizado no mundo para a abordagem multidimensional de problemas relacionados ao abuso de SPA é a Escala de Gravidade de Dependência (Addiction Severity Index, ASI).

Objetivo: Através da revisão da literatura sobre o tema, da apresentação de um caso clínico, de aspectos da ASI e da discussão de tópicos contemplados na avaliação de usuários de drogas, este trabalho tem como finalidade auxiliar o profissional de saúde a sistematizar a avaliação desses pacientes.

Método: A revisão da literatura foi realizada nas bases de dados MEDLINE, LILACS e PsycINFO, utilizando os descritores assessment e evaluation, cruzados com o termo substance-related disorders dos Medical Subject Headings e subexpressões nele incluídas.

Resultados e conclusão: Na literatura, há uma carência de artigos sobre o tema, e, no Brasil, ainda não existem instrumentos que se proponham a avaliar os usuários de drogas de forma tão ampla como a ASI. A utilização de uma escala com essas características pode ser útil para o sistema de saúde brasileiro, favorecendo a identificação precoce de problemas e propiciando melhora na qualidade da assistência prestada.

Descritores: Avaliação, alcoolismo, transtornos por uso de substâncias.

\begin{abstract}
Introduction: Before initiating any treatment for substance abuse, the assessment of problems related to the consumption of those substances is of paramount importance. Considering the complexity of this evaluation, the instrument most widely used in the present days for a multidimensional approach to these patients is the Addiction Severity Index (ASI).

Objective: The present study presents a literature review, a clinical case vignette, comments on different aspects of the scale, and the discussion of topics covered in the evaluation process, and aims at providing support to health professionals in the sense of systematizing the assessment of these patients.

Methods: The literature review was conducted on MEDLINE, LILACS, and PsycINFO databases, using the keywords assessment and evaluation together with substance-related disorders and other terms presented as synonyms in the Medical Subject Headings.

Results and conclusion: There is a paucity of articles in the literature focusing on the topic, and no other instruments designed to provide a general overview of substance users (as is the case with the ASI) are available in Brazil. The use of a scale with these characteristics may be useful for the Brazilian public health system, allowing for the early identification of problems and promoting an improvement in the quality of treatment provided to these patients.
\end{abstract}

Keywords: Diagnosis of health situation, alcoholism, substance-related disorders.

Correspondência:

Felix Henrique Paim Kessler, Rua Itaqui, 89/103, CEP 90460-140, Porto Alegre, RS. E-mail: kessler@ez-poa.com.br

Não foram declarados conflitos de interesse associados à publicação deste artigo.

Copyright (C) Revista de Psiquiatria do Rio Grande do Sul - APRS

Recebido em 14/12/2009. Aceito em 19/01/2010. 


\section{Introdução}

Avanços recentes têm contribuído para aumentar o conhecimento referente à temática do consumo de substâncias psicoativas (SPA). Tornou-se evidente que, antes de iniciar um tratamento, além de investigar um possível diagnóstico de abuso e dependência, é primordial avaliar as características do uso da droga, tais como frequência, quantidade e duração, assim como os prejuízos que ela acarreta à vida dos usuários. $\mathrm{O}$ conceito de avaliação nesse campo se expandiu, considerando sua complexidade, e passou a incluir outros aspectos igualmente importantes da vida do indivíduo. Problemas clínicos, como dores crônicas, por exemplo, podem ser causados ou exacerbados pelo uso de drogas, assim como o uso das mesmas pode ser fonte de alívio dessas dores. Achados como esse geraram uma série de hipóteses etiológicas, como a denominada "teoria da automedicação", que propõe que o ambiente sociocultural e os relacionamentos interpessoais influenciam claramente o uso drogas, buscando, através dos seus efeitos, um melhor equilíbrio do "meio interno".

Nesse contexto, foi desenvolvido por McLellan et al., um instrumento para a abordagem multidimensional das questões relacionadas ao abuso de SPA, incluindo a gravidade e a necessidade de tratamento para os problemas clínicos e psicossociais. A Escala de Gravidade de Dependência (Addiction Severity Index, ASI) ${ }^{2,3}$ consiste em uma entrevista semiestruturada que proporciona uma avaliação global da situação atual (últimos 30 dias) e passada (6 meses) do paciente, ponderando a gravidade dos problemas em sete áreas: médica, ocupacional, aspectos legais, sociofamiliares, psiquiátricos, uso de álcool e uso de outras drogas. Recentemente, a sexta versão da ASI foi adaptada para o contexto brasileiro (detalhes do processo podem ser encontrados no artigo de adaptação ${ }^{4}$ ) e submetida a um estudo de validação, visto que o país ainda necessita de protocolos mais completos de avaliação dirigidos aos usuários de SPA no sistema público de saúde ${ }^{5}$. O instrumento, em português, juntamente com seu manual de; aplicação, encontram-se disponíveis nos sites www.cpad.org.br e www.obid.senad.gov.br.

Este artigo tem a finalidade de auxiliar o profissional de saúde a sistematizar e melhorar o atendimento desses pacientes na prática clínica. Serão descritos um caso clínico e aspectos importantes da própria ASI, que servirão como guia para ilustrar os tópicos contemplados na avaliação do paciente.

\section{Método}

Foi realizada uma revisão dos estudos relacionados à avaliação de usuários de álcool e outras SPA, identificados nas bases de dados internacionais MEDLINE, LILACS e PsycINFO. Para tanto, foram selecionados os descritores assessment e evaluation. Essas palavras-chave foram cruzadas com o termo substance-related disorders, extraído dos Medical Subject Headings (MeSH), que engloba as expressões drug dependence, drug addiction, drug abuse, drug use disorder, substance use disorders, substance abuse, substance dependence e substance addiction.

Foram considerados estudos publicados nos idiomas português, espanhol e inglês, e foram excluídos estudos de avaliação de serviços para o dependente químico, de validação e avaliação psicométrica de instrumentos, entrevistas e estudos realizados com crianças e adolescentes; capítulos de livros e artigos não indexados também foram usados como forma de complementação.

\section{Resultados}

Foram encontrados 5.357 estudos contendo os descritores no título do artigo. Do total, somente 68 foram incluídos na revisão após leitura do resumo, por versarem especificamente sobre a importância da avaliação ou sobre como avaliar os referidos aspectos no usuário de SPA.

As primeiras publicações sobre como avaliar um usuário de álcool e outras drogas ${ }^{6-19}$ datam da década de 1960, porém apenas a partir dos anos 1990 é que os elementos essenciais relacionados à dependência química foram melhor elucidados e organizados ${ }^{20-56}$. A maioria dos estudos, entretanto, refere-se a populações específicas, não abordando a questão de forma mais ampla, didática e sistematizada ${ }^{57-67}$.

A seguir são descritos os achados referentes aos elementos essenciais da avaliação do usuário de SPA, ilustrados pela apresentação de um caso clínico.

\section{Apresentação de vinheta clínica}

Aos 48 anos, João (nome fictício) foi encaminhado para sua segunda internação devido ao uso problemático de álcool e cocaína. Ele é zelador, casado e pai de dois adolescentes. Fuma 20 cigarros por dia há 30 anos e consome bebidas alcoólicas há 20 anos, mas de forma mais intensa nos últimos 10 anos. Atualmente, ingere aproximadamente uma garrafa de cachaça por dia, quatro dias por semana. Bebe a maior parte do dia, aborrecendo-se quando não pode beber. Iniciou o uso de cocaína inalada há dois anos, pois assim "conseguia energia para trabalhar". Em seu tratamento ambulatorial mais recente para o abuso de SPA, João foi diagnosticado como dependente de álcool, cocaína e nicotina. No início do presente tratamento, João foi submetido a uma avaliação multidimensional na qual a ASI foi utilizada como base.

\section{A ASI}

Normalmente, a ASI é utilizada no início do tratamento, e seus escores fornecem um perfil geral da gravidade dos problemas em cada área específica, o que torna seu uso altamente relevante para a prática clínica, especialmente no planejamento do tratamento, avaliação dos resultados e encaminhamento do paciente. 
O instrumento possui uma complexa estrutura de resposta, por englobar questões de vários formatos relativos ao período no tempo (presente e passado) e ao tipo de informação obtida. Seu diferencial está no uso das avaliações da gravidade dos problemas em cada subárea, além do seu respectivo escore sumário. Estes últimos foram baseados em métodos racionais e empíricos, desenvolvidos a partir de combinações aritméticas e outros cálculos matemáticos envolvendo os itens estatisticamente mais relevantes de cada área $^{2}$. Por sua vez, a gravidade dos problemas, que é baseada no período de 30 dias anterior à avaliação, proporciona uma estimativa do estado atual do paciente, de acordo com sua própria percepção. Apesar de a gravidade de dependência ser avaliada conforme os critérios diagnósticos estabelecidos em manuais como o Diagnostic and Statistical Manual of Mental Disorders, $4^{\mathrm{a}}$ edição (DSM-IV) e a Classificação Internacional de Doenças, $10^{\mathrm{a}}$ edição (CID-10), na ASI ela é avaliada com perguntas sobre o grau de preocupação do paciente em relação à sua dificuldade e à necessidade de tratamento para aquela área.

Os autores da versão original do instrumento sugerem que os entrevistadores sejam profissionais da saúde e que, além da leitura do manual, realizem um treinamento com investigadores com mais experiência na aplicação do instrumento*. Deve-se respeitar o formato da entrevista, uma vez que este garante sua validade e confiabilidade.

Para padronizar a avaliação subjetiva e a comunicação do próprio paciente sobre a extensão dos problemas experimentados em cada uma das áreas do instrumento, é empregada uma escala de 5 pontos (0-4), sendo que os pacientes podem graduar a gravidade de seus problemas mostrando seu grau de preocupação e até que ponto eles sentem que o tratamento é importante, da seguinte forma: 0 - nada, 1 - levemente, 2 - moderadamente, 3 - consideravelmente e 4 - extremamente.

Assim, é oferecida ao paciente a oportunidade de manifestar sua verdadeira opinião, e isso deve ser buscado também pelo entrevistador, que deverá manifestar imparcialidade na condução da entrevista, não impondo suas próprias percepções e respostas. No que diz respeito à abordagem e ao modo de realizar as questões, caso o paciente não compreenda a pergunta após a leitura literal, pode-se comunicar sua ideia-chave fazendo uso de sinônimos, de acordo com o nível de escolaridade e compreensão do paciente.

\section{Relação terapêutica/vínculo}

Quando João chegou ao tratamento, demonstrou uma compreensão pobre sobre os prejuízos relacionados ao consumo de bebidas alcoólicas e de cocaína, apresentando-se um pouco resistente a qualquer intervenção no sentido de investigar padrões de uso. Afirmava que não percebia o uso dessas substâncias como seu maior problema, mas sim seu modo de lidar com as dificuldades impostas pelo trabalho: insistia que, se pudesse ficar afastado do serviço por um período de 15 dias, com certeza apresentaria melhoras em sua saúde.

A ambivalência quanto ao uso de SPA é comum no início do tratamento, e muitos pacientes só vão à consulta por imposição de familiares, amigos ou do sistema judiciário ${ }^{68}$. A opção mais adequada, no início, pode ser manter o foco na redução da ansiedade do paciente, realizando entrevistas com perguntas abertas e não confrontativas. Em alguns casos, é preciso postergar o uso de protocolos estruturados de avaliação. Um vínculo terapêutico genuíno estabelecido com o paciente e um contrato de comum acordo entre as partes servem como base para o tratamento, podendo garantir sua continuidade e qualidade. Essa abordagem diminui resistên$\operatorname{cias}^{69} \mathrm{e}, \mathrm{com}$ isso, aumenta a motivação do paciente para cooperar e participar do que está sendo proposto ${ }^{70}$.

João foi informado de que o objetivo da avaliação era obter informações que subsidiassem futuras intervenções para que houvesse uma melhora na qualidade de sua vida e saúde. Foi encorajado a falar sobre o que considerava serem seus problemas atuais, assim como as fontes de prazer, aproveitamento de seu tempo livre e sua rotina, sem perguntas diretas sobre abuso de SPA. Tal estratégia permitiu ao paciente sentir-se mais à vontade em relação ao processo terapêutico.

\section{Anamnese}

Os principais objetivos de uma anamnese são: favorecer o engajamento ou a adesão ao tratamento; colher dados sobre o histórico da evolução do consumo e de como a droga influenciou os problemas apresentados na vida do indivíduo; identificar fatores que favoreceram a instalação do uso e mantêm a dependência; identificar fatores que favorecem a abstinência; e reunir informações para estabelecer hipóteses diagnósticas ${ }^{71}$.

Um levantamento completo e detalhado da história do indivíduo é necessário para avaliar a gravidade da dependência bem como do comprometimento dos aspectos físicos, psicológicos e sociais de sua vida, fundamentais para determinar o tipo de tratamento mais adequado. Investigar aspectos da personalidade, crenças e valores pessoais do paciente, antes e depois do início do abuso de drogas, contribui para uma visão geral da pessoa e do seu estilo de interação com o mundo e com os demais. Comumente, a fim de complementar a avaliação e obter informações de forma mais objetiva e completa, deve-se solicitar entrevistas com familiares.

\section{Problemas clínicos}

É conhecida a relação entre o uso de álcool e drogas e uma vasta gama de complicações clínicas, como doenças car-

\footnotetext{
* Atualmente, existem alguns centros de pesquisa no Brasil (citados no manual) capacitados para realizar o treinamento para a aplicação da ASI. Esse treinamento não é obrigatório, mas fortemente recomendável, especialmente no caso de investigações científicas. Os interessados no treinamento podem entrar em contato com um desses centros.
} 
díacas, hepáticas, renais, respiratórias, gastrointestinais e neurológicas. Além disso, uma série de outras doenças vinculadas a comportamentos de risco devido a esse consumo também podem ocorrer, por exemplo AIDS e outras doenças sexualmente transmissíveis ${ }^{72-75}$. A ASI aborda as principais doenças relacionadas de forma direta ao uso de álcool e outras SPA.

João, ao ser perguntado sobre a frequência e a gravidade de sintomas físicos no mês prévio à avaliação, relatou sentir dores na região torácica e apresentar sintomas de complicações gástricas, as quais, posteriormente, revelaram uma gastrite não infecciosa.

\section{Problemas legais}

Apesar de ser um item frequentemente desprezado em avaliações de dependentes químicos, a investigação de problemas com a justiça e participação em atividades ilegais fazse muito necessária ${ }^{76,77}$. Essas dificuldades exercem influência em vários âmbitos da vida, prejudicando sua qualidade e ameaçando a integridade física e psicológica do indivíduo e dos demais. A ASI tem papel facilitador para que esse tema venha a ser discutido, sendo essencial que, durante sua aplicação, o tom não preconceituoso e a imparcialidade predominem.

Antes da aplicação do instrumento, João nada havia comentado sobre problemas legais. A partir das respostas fornecidas na ASI, constatou-se que o paciente havia sido denunciado duas vezes pela ex-mulher: os processos judiciais acarretaram ao paciente alguns meses de detenção.

\section{Problemas sociofamiliares}

É muito improvável que o uso abusivo de alguma SPA não cause algum grau de impacto na família do usuário. A violência é uma das consequências mais nocivas que uma família pode experimentar, sendo as crianças e os adolescentes especialmente vulneráveis a ela ${ }^{78}$. Em três áreas da ASI (problemas legais, familiares e psiquiátricos), são investigados indícios de violência e eventos traumáticos. Quando há dúvidas e/ou incoerências, estas devem ser objeto de esclarecimento, sejam elas referentes à família ou a outras relações (amigos, por exemplo).

As perguntas realizadas nessas três sessões ajudaram a coletar informações complementares sobre o comportamento impulsivo de João e sobre sua interação com os filhos e com sua ex-mulher. O paciente já havia comentado, na área legal, que sofrera detenções devido ao não pagamento da pensão alimentícia, o que deixou o avaliador mais atento às perguntas sobre os filhos na área das relações familiares. João havia, também, agredido a ex-mulher e tinha relação distante com os filhos, situação agravada após sua detenção.

\section{Escolaridade, emprego, renda e sustento}

Atualmente, é consenso na literatura científica que o uso de SPA é responsável por casos de baixo rendimento e absenteísmo no trabalho. Entretanto, muitas vezes a relação é inversa, e as más condições ocupacionais tornam o ambiente hostil, de modo que o funcionário pode buscar, no uso de álcool ou outras substâncias, um alívio para esse desconforto. A relação do paciente com outras formas de adição, como o jogo patológico, deve ser avaliada, pois frequentemente acarreta dificuldades financeiras ao indivíduo.

João trabalha há 28 anos no mesmo local, exercendo a mesma função. Interrompeu seus estudos aos 14 anos e, em sua vida inteira, teve somente dois empregos na mesma área, não possuindo diploma de qualquer outro curso ou treinamento. Seu cargo como zelador obrigava-o a residir no local, onde acabava envolvido 24 horas por dia: por falta de mão de obra, cumpria atribuições de outros profissionais. João considerou extremamente importante receber orientação para lidar com problemas profissionais ao longo do tratamento, uma vez que não se sentia capaz de resolver os conflitos atuais nem de procurar outro trabalho. Conforme a história relatada pelo paciente, ficou clara a relação entre os problemas profissionais e o agravamento do uso de álcool e cocaína.

\section{Qualidade de vida e atividades de lazer}

Em pacientes com abuso ou dependência de álcool, a qualidade de vida está mais correlacionada aos prejuízos relacionados ao consumo do que ao seu padrão (frequência e quantidade de uso). Um estudo brasileiro, por exemplo, demonstrou que a gravidade da dependência pode ser um importante indicativo de diminuição na qualidade de vida ${ }^{79,80}$.

Durante a aplicação da ASI, foi constatado que João não participava de nenhuma atividade religiosa e/ou de lazer. De acordo com sua própria percepção, apresentava dificuldade em aproveitar momentos destinados ao descanso, estando sempre sob o efeito do uso de alguma substância: consumia bebidas alcoólicas para conseguir relaxar, descansar e desempenhar suas funções; no momento de voltar ao trabalho, usava cocaína.

\section{Comorbidades psiquiátricas}

As comorbidades psiquiátricas são muito prevalentes em usuários de álcool e outras $\mathrm{SPA}^{81-86}$, sendo capazes de mascarar a sintomatologia comumente encontrada em dependentes químicos. Além disso, geralmente interferem no plano de tratamento, influenciando a prescrição de psicofármacos e a indicação do tipo de psicoterapia ${ }^{87-89}$. A impulsividade se configura como característica comum a vários transtornos psiquiátricos associados à dependência química, merecendo avaliação específica por contribuir para o aumento do número de recaídas e da exposição a situações de risco ${ }^{90}$.

Através das respostas à área psiquiátrica da ASI, a equipe tomou conhecimento de que João nunca havia realizado alguma avaliação que contemplasse problemas psicológicos e psiquiátricos. No entanto, afirmou perceber indicativos de impulsividade e episódios de alterações de humor, relatando dificuldades no controle de seu temperamento e períodos prolongados de ansiedade e tristeza, mesmo em momentos da 
vida em que o abuso de drogas não estava presente. Posteriormente, esses sintomas foram melhor investigados e foi confirmado o diagnóstico de transtorno de estresse pós-traumático, desencadeado após sua prisão e que ele relacionava ao aumento do uso de álcool, e de transtorno do humor bipolar.

\section{Resultados e plano terapêutico}

O plano terapêutico de João foi elaborado de acordo com os resultados da aplicação da ASI (escores de gravidade). Seu resumo é apresentado na Tabela 1 .

Tabela 1 - Resultado dos escores de gravidade

\begin{tabular}{|c|c|c|c|c|}
\hline Subescalas & Questão & $\begin{array}{c}\text { Grau de } \\
\text { preocupação }\end{array}$ & Questão & $\begin{array}{l}\text { Necessidade de } \\
\text { tratamento }\end{array}$ \\
\hline Drogas & $\begin{array}{l}\text { Nos últimos } 30 \text { dias, quão } \\
\text { preocupado ou incomodado você } \\
\text { tem estado com esses problemas } \\
\text { com drogas? }\end{array}$ & 4 & $\begin{array}{l}\text { Neste momento, quão importante } \\
\text { é para você o tratamento (atual ou } \\
\text { adicional) para o seu uso de drogas? }\end{array}$ & 4 \\
\hline $\begin{array}{l}\text { Família/ } \\
\text { Social }\end{array}$ & $\begin{array}{l}\text { Nos últimos } 30 \text { dias, quão } \\
\text { preocupado ou incomodado } \\
\text { você tem estado com quaisquer } \\
\text { problemas com os seus } \\
\text { relacionamentos com adultos? }\end{array}$ & 4 & $\begin{array}{l}\text { Neste momento, quão importante } \\
\text { é para você receber um auxílio, } \\
\text { aconselhamento ou tratamento (atual } \\
\text { ou adicional) para seus problemas } \\
\text { de relacionamento com adultos? }\end{array}$ & 4 \\
\hline Lazer & $\begin{array}{l}\text { Quão satisfeito você tem estado com } \\
\text { a forma com que você aproveita o } \\
\text { seu tempo livre? }\end{array}$ & $0 *$ & --- & --- \\
\hline $\begin{array}{l}\text { Emprego/ } \\
\text { Sustento }\end{array}$ & --- & --- & $\begin{array}{l}\text { Neste momento, quão importante é } \\
\text { para você receber qualquer tipo de } \\
\text { orientação (como aconselhamento, } \\
\text { treinamento ou educação) para } \\
\text { ajudá-lo a se preparar para ou a } \\
\text { encontrar um emprego, ou lidar com } \\
\text { problemas profissionais? }\end{array}$ & 4 \\
\hline Trauma & $\begin{array}{l}\text { Nos últimos } 30 \text { dias, quão } \\
\text { preocupado ou incomodado você } \\
\text { tem estado com sentimentos, } \\
\text { pensamentos ou outras reações } \\
\text { relacionadas a esses eventos? }\end{array}$ & 3 & $\begin{array}{l}\text { Neste momento, quão importante } \\
\text { é para você receber auxílio, } \\
\text { aconselhamento ou tratamento } \\
\text { (atual ou adicional) para quaisquer } \\
\text { sentimentos, pensamentos ou } \\
\text { outras reações relacionadas a esses } \\
\text { eventos? }\end{array}$ & 3 \\
\hline Psiquiátrica & $\begin{array}{l}\text { Quão preocupado ou incomodado } \\
\text { você tem estado com esses } \\
\text { problemas psicológicos ou } \\
\text { psiquiátricos? }\end{array}$ & 3 & $\begin{array}{l}\text { Neste momento, quão importante } \\
\text { é para você o tratamento (atual } \\
\text { ou adicional) para problemas } \\
\text { psicológicos/psiquiátricos? }\end{array}$ & 3 \\
\hline Médica & $\begin{array}{l}\text { Quão preocupado ou incomodado } \\
\text { você tem estado com sua saúde } \\
\text { física ou qualquer problema clínico? }\end{array}$ & 3 & $\begin{array}{l}\text { Neste momento, quão importante } \\
\text { é para você o tratamento (atual ou } \\
\text { adicional) para qualquer problema } \\
\text { clínico ou físico? }\end{array}$ & 3 \\
\hline Álcool & $\begin{array}{l}\text { Nos últimos } 30 \text { dias, quão } \\
\text { preocupado ou incomodado você } \\
\text { tem estado com esses problemas } \\
\text { com álcool? }\end{array}$ & 2 & $\begin{array}{l}\text { Neste momento, quão importante } \\
\text { é para você o tratamento (atual ou } \\
\text { adicional) para o seu uso de álcool? }\end{array}$ & 2 \\
\hline Legal & $\begin{array}{l}\text { Quão graves você considera seus } \\
\text { problemas atuais com a justiça } \\
\text { criminal? }\end{array}$ & 0 & --- & --- \\
\hline
\end{tabular}

* Esse escore específico não reflete o grau de preocupação do paciente em relação ao lazer, e sim a satisfação com a forma como o tempo livre é aproveitado. 
Após um cuidadoso feedback da avaliação realizada, partindo de suas próprias respostas, João pareceu compreender que seus problemas estavam inter-relacionados e que não havia somente um comportamento ou aspecto da vida que estava disfuncional. O consumo de bebidas alcoólicas, assim como o abuso de cocaína, que eram vistos inicialmente como um apoio para poder enfrentar as dificuldades com o trabalho, gradualmente foram sendo percebidos como uma estratégia pouco eficaz que desencadeava e agravava a maioria dos problemas apresentados. Assim, a ASI também foi utilizada para motivar João para o tratamento, pois oportunizou a organização de informações relevantes, mostrando, sob a forma de resultados objetivos - os escores de gravidade -, informações subjetivas relatadas pelo próprio paciente.

Como se pode observar na Tabela 1, as áreas de maior preocupação à época da avaliação eram uso de SPA, família, lazer e emprego. João considerou ser muito importante um aconselhamento para ajudá-lo a lidar com problemas ocupacionais, no intuito de encontrar outro emprego. Em segundo lugar, os problemas com a família eram os que mais o preocupavam.

Concluiu-se que a violência doméstica era uma constante em sua vida conjugal. Na questão referente à forma de aproveitar o tempo livre, João referiu não encontrar nenhuma satisfação nos períodos de folga e atividades de lazer. Em relação ao uso de cocaína, disse estar extremamente preocupado em como enfrentar a intensa compulsão, afirmando ser extremamente importante desenvolver uma estratégia especial para esse problema.

Como o prognóstico inicial do paciente era reservado, devido à gravidade dos problemas, foi sugerido que fossem incluídos no plano terapêutico todos os aspectos descritos anteriormente, através de uma abordagem multidisciplinar.

Inicialmente, o paciente permaneceu em regime de internação durante um período de 30 dias, para, posteriormente, seguir tratamento ambulatorial com frequência mínima de duas vezes por semana.

João recebeu uma abordagem psicoeducacional e motivacional para reforçar a implicação da ingestão do álcool em sua saúde física, sua problemática ocupacional e a relação com seu uso de cocaína. Além disso, ele foi orientado sobre a compulsão e os sintomas de abstinência, o que serviu para reduzir sua ansiedade e aumentar a sua autoeficácia. Foram trabalhadas, também, estratégias de prevenção de recaída, de enfrentamento e resolução de problemas. Como complemento, João frequentou grupos de autoajuda durante 4 meses.

Os problemas ocupacionais mereceram um tópico especial no planejamento do tratamento, como foi solicitado pelo paciente. Foi acordado com seu superior uma diminuição de atribuições no trabalho e delimitado um horário fixo para a realização de suas tarefas. A família de João concordou em participar efetivamente do tratamento através de sessões de terapia familiar.

Após 3 meses de tratamento ambulatorial, João foi submetido a uma nova avaliação através da ASI (Tabela 2), para fins de acompanhamento e avaliação do tratamento ${ }^{3}$.
Na Tabela 2, pode-se observar que João reduziu seu grau de preocupação nas subescalas Drogas, Família/Social e Emprego e aumentou seu grau de satisfação com suas atividades de lazer. Como o paciente apresentou progressos significativos, a frequência de sessões de psicoterapia foi reduzida para um encontro por semana.

É importante salientar que este artigo não tem o objetivo de sugerir ou testar a eficácia do tratamento descrito, mas apenas ilustrar como ele pode ser avaliado através da escala. Além disso, ressalta-se também a possibilidade de utilizar o chamado escore sumário das seções, que é calculado a partir desses escores subjetivos e de outras questões objetivas. Esse tema será enfocado no artigo sobre a validação brasileira do instrumento que está sendo preparado para publicação por este mesmo grupo de autores.

\section{Discussão}

$\mathrm{Na}$ literatura nacional e internacional, há uma lacuna referente à descrição do exame conjunto dos problemas de usuários de álcool e outras SPA. Apesar da existência de outras escalas de avaliação, não há nenhuma entrevista adaptada para a cultura brasileira que se proponha a avaliar esses problemas de forma tão ampla como a ASI. A disponibilidade de um instrumento de avaliação com essas características é de grande valia, pela facilidade e possibilidade de padronização das informações.

Instrumentos como a ASI podem ser muito úteis tanto na pesquisa quanto na avaliação clínica. Para evitar que seu uso se torne impessoal, o avaliador deve utilizar sua experiência e empatia, considerando a individualidade do paciente e suas opiniões sobre o tratamento e o uso de SPA. O grande diferencial e ponto positivo da ASI em relação à maioria dos instrumentos de avaliação disponíveis são os escores subjetivos que avaliam a gravidade dos problemas, uma vez que, ao considerar a percepção do próprio indivíduo, permitem ao avaliador definir os objetivos do tratamento de acordo com os interesses do paciente, não se afastando do foco predominante de cuidado para com ele. Há algumas limitações do instrumento, como a falta de perguntas sobre sexualidade, por exemplo, e o tempo de entrevista, que costuma ser longo. Contudo, se, por um lado, a ASI não se destina à triagem de pacientes, por outro ela permite ir além da avaliação e acompanhar o paciente em diversas fases do tratamento ${ }^{91}$.

Segundo a opinião destes autores, o uso da ASI poderia ser útil para o sistema de saúde brasileiro, auxiliando na avaliação de dependentes químicos. Existe ainda a possibilidade do desenvolvimento de uma versão mais compacta do instrumento, que poderia ser aplicada mais rapidamente. Seu uso propiciaria uma melhora na qualidade da assistência prestada, favorecendo a identificação precoce de problemas e evitando encaminhamentos equivocados. Facilitaria, também, o desenvolvimento de planos de tratamento que contemplassem as complexas necessidades desses pacientes. 
Tabela 2 - Resultado dos escores de gravidade após 3 meses de tratamento

\begin{tabular}{|c|c|c|c|c|}
\hline Subescalas & Questão & $\begin{array}{c}\text { Grau de } \\
\text { preocupação }\end{array}$ & Questão & $\begin{array}{l}\text { Necessidade de } \\
\text { tratamento }\end{array}$ \\
\hline Drogas & $\begin{array}{l}\text { Nos últimos } 30 \text { dias, quão } \\
\text { preocupado ou incomodado você } \\
\text { tem estado com esses problemas } \\
\text { com drogas? }\end{array}$ & 2 & $\begin{array}{l}\text { Neste momento, quão importante } \\
\text { é para você o tratamento (atual ou } \\
\text { adicional) para o seu uso de drogas? }\end{array}$ & 2 \\
\hline $\begin{array}{l}\text { Família/ } \\
\text { Social }\end{array}$ & $\begin{array}{l}\text { Nos últimos } 30 \text { dias, quão } \\
\text { preocupado ou incomodado } \\
\text { você tem estado com quaisquer } \\
\text { problemas com os seus } \\
\text { relacionamentos com adultos? }\end{array}$ & 2 & $\begin{array}{l}\text { Neste momento, quão importante } \\
\text { é para você receber um auxílio, } \\
\text { aconselhamento ou tratamento (atual } \\
\text { ou adicional) para seus problemas } \\
\text { de relacionamento com adultos? }\end{array}$ & 2 \\
\hline Álcool & $\begin{array}{l}\text { Nos últimos } 30 \text { dias, quão } \\
\text { preocupado ou incomodado você } \\
\text { tem estado com esses problemas } \\
\text { com álcool? }\end{array}$ & 2 & $\begin{array}{l}\text { Neste momento, quão importante } \\
\text { é para você o tratamento (atual ou } \\
\text { adicional) para o seu uso de álcool? }\end{array}$ & 2 \\
\hline Psiquiátrica & $\begin{array}{l}\text { Quão preocupado ou incomodado } \\
\text { você tem estado com esses } \\
\text { problemas psicológicos ou } \\
\text { psiquiátricos? }\end{array}$ & 2 & $\begin{array}{l}\text { Neste momento, quão importante } \\
\text { é para você o tratamento (atual } \\
\text { ou adicional) para problemas } \\
\text { psicológicos/psiquiátricos? }\end{array}$ & 2 \\
\hline $\begin{array}{l}\text { Emprego/ } \\
\text { Sustento }\end{array}$ & --- & --- & $\begin{array}{l}\text { Neste momento, quão importante é } \\
\text { para você receber qualquer tipo de } \\
\text { orientação (como aconselhamento, } \\
\text { treinamento ou educação) para ajudá- } \\
\text { lo a se preparar para ou a encontrar } \\
\text { um emprego, ou lidar com problemas } \\
\text { profissionais? }\end{array}$ & 2 \\
\hline Médica & $\begin{array}{l}\text { Quão preocupado ou incomodado } \\
\text { você tem estado com sua saúde } \\
\text { física ou qualquer problema clínico? }\end{array}$ & 1 & $\begin{array}{l}\text { Neste momento, quão importante } \\
\text { é para você o tratamento (atual ou } \\
\text { adicional) para qualquer problema } \\
\text { clínico ou físico? }\end{array}$ & 1 \\
\hline Trauma & $\begin{array}{l}\text { Nos últimos } 30 \text { dias, quão } \\
\text { preocupado ou incomodado você } \\
\text { tem estado com sentimentos, } \\
\text { pensamentos ou outras reações } \\
\text { relacionadas a esses eventos? }\end{array}$ & 1 & $\begin{array}{l}\text { Neste momento, quão importante } \\
\text { é para você receber auxílio, } \\
\text { aconselhamento ou tratamento } \\
\text { (atual ou adicional) para quaisquer } \\
\text { sentimentos, pensamentos ou } \\
\text { outras reações relacionadas a esses } \\
\text { eventos? }\end{array}$ & 1 \\
\hline Lazer & $\begin{array}{l}\text { Quão satisfeito você tem estado com } \\
\text { a forma com que você aproveita o } \\
\text { seu tempo livre? }\end{array}$ & $3 *$ & --- & --- \\
\hline Legal & $\begin{array}{l}\text { Quão graves você considera seus } \\
\text { problemas atuais com a justiça } \\
\text { criminal? }\end{array}$ & 0 & --- & --- \\
\hline
\end{tabular}

* Esse escore específico não reflete o grau de preocupação do paciente em relação ao lazer, e sim a satisfação com a forma como o tempo livre é aproveitado. 


\section{Referências}

1. Khantzian EJ. The self-medication hypothesis of addictive disorders: focus on heroine and cocaine dependence. Am J Psychiatry. 1985;142:1259-64.

2. McLellan A, Luborsky L, Woody G, OBrien C. An improved diagnostic evaluation instrument for substance abuse patients. The Addiction Severity Index. J Nerv Ment Dis. 1980;168(1):26-33.

3. McLellan AT, Cacciola J, Alterman A, Rikoon S, Carise D. The Addiction Severity Index at 25: origins, contributions and transitions. Am J Addict. 2006;15(2):113-24.

4. Kessler FHP. Adaptação transcultural multicêntrica da sexta versão da Escala de Gravidade de Dependência (ASI6) para o Brasil. Rev Psiquiatr Rio Gd Sul. 2007;29(3):335-6

5. Kessler F, Woody G, De Boni R, Von Diemen L, Benzano D, Faller S, et al. Evaluation of psychiatric symptoms in cocaine users in the Brazilian public health system: need for data and structure. Public Health. 2008;122(12):1349-55.

6. Paton W. Drug dependence: a socio-pharmacological assessment. Adv Sci. 1968;25(124):200-12.

7. Louria D. Drug abuse: a current assessment. Am Fam Physician GP. 1970;1(6):74-80.

8. Morselli P, Placidi G, Maggini C, Gomeni R, Guazelli M, De Lisio G, et al. An integrated approach for the evaluation of psychotropic drug in man. I. Studies on amphetamine. Relationship between drug levels and psychophysiological measurements. Psychopharmacologia. 1976;46(2):211-7.

9. Carroll J, Malloy T, Kenrick F. Drug abuse by alcoholics and problem drinkers: a literature review and evaluation. Am J Drug Alcohol Abuse. 1977;4(3):317-41.

10. Holsten F, Waal H. Evaluation of the drug taker. A step to an evaluation of treatment. Acta Psychiatr Scand Suppl. 1980;284:29-41

11. Gitlow S. Considerations on the evaluation and treatment of substance dependency. J Subst Abuse Treat. 1985;2(3):175-9.

12. Stacy AW, Widaman KF, Hays R, DiMatteo MR. Validity of self-reports of alcohol and other drug use: a multitrait-multimethod assessment. J Pers Soc Psychol. 1985;49(1):219-32.

13. Castillo Pérez P. [Ethical aspects of drug evaluation in human beings]. Rev Clin Esp. 1986;179(1):42-9.

14. Hasin D, Grant B. Assessment of specific drug disorders in a sample of substance abuse patients: a comparison of the DIS and the SADS-L procedures. Drug Alcohol Depend. 1987;19(2):165-76.

15. Stein J, Newcomb M, Bentler P. Structure of drug use behaviors and consequences among young adults: multitrait-multimethod assessment of frequency, quantity, work site, and problem substance use. J Appl Psychol. 1988;73(4):595-605.

16. Sweeney J, Meisel L, Walsh V, Castrovinci D. Assessment of cognitive functioning in poly-substance abusers. J Clin Psychol. 1989;45(2):346-51

17. Richardson H. Volatile substance abuse: evaluation and treatment. Hum Toxicol. 1989;8(4):319-22

18. Nuckols C, Greenson J. Cocaine addiction. Assessment and intervention. Nurs Clin North Am. 1989;24(1):33-43

19. Kauffman J, Shaffer H, Burglass M. A strategy for the biological assessment of addiction. Adv Alcohol Subst Abuse. 1983-1984 Fall-Winter;3(1-2):7-18.

20. Gaulier B, Butler S, Haller D. Problems associated with the assessment of personality disorders in substance abusers. NIDA Res Monogr. 1990;105:599-600.

21. Fagerstrom K, Heatherton T, Kozlowski L. Nicotine addiction and its assessment. Ear Nose Throat J. 1990;69(11):763-5.

22. McLellan A, Randall M, Joseph N, Alterman A. Categorizing substance abusers using the ASI: implications for evaluation and treatment. NIDA Res Monogr. 1990;105:227-35.

23. Butler S, Gaulier B, Haller D. Assessment of axis II personality disorders among female substance abusers. Psychol Rep. 1991;68(3 Pt 2):1344-6.

24. Bigelow G. Human drug abuse liability assessment: opioids and analgesics. Br J Addict. 1991;86(12):1615-23.

25. Lisanti P. Assessment of the adult client for drug and alcohol use. NLN Publ. 1991(15-2407):151-247.

26. OHare $\mathrm{T}$. The substance-abusing chronically mentally ill client: prevalence, assessment, treatment, and policy concerns. Soc Work. 1992;37(2):185-7.

27. Burns C. Assessment and screening for substance abuse: guidelines for the primary care nurse practitioner. Nurse Pract Forum. 1993;4(4):199-206.

28. Curtiss M, Lenz K, Frei N. Medical evaluation of African American women entering drug treatment. J Addict Dis. 1993;12(4):29-44.

29. Sees K, Clark H. Opioid use in the treatment of chronic pain: assessment of addiction. J Pain Symptom Manage. 1993;8(5):257-64.

30. Simeone R, Frank B, Aryan Z. Needs assessment in substance misuse: a comparison of approaches and case study. Int J Addict. 1993;28(8):767-92.

31. 33rd Annual Meeting of the New Clinical Drug Evaluation Unit (NCDEU). Boca Raton, Florida, June 1993. Proceedings and abstracts. Psychopharmacol Bull. 1994;30(1):1-141.
32. Altimir M, Serras E, Torrens M. [Detection and evaluation of drug addict patients seen at a primary care center]. Aten Primaria. 1994;13(5):252-4.

33. Frances R. Substance abuse in the general hospital. A priority area for evaluation and treatment. Gen Hosp Psychiatry. 1994;16(2):71-2.

34. Moore R. Screening and assessment of alcohol and other drug abuse. Md Med J. 1994;43(1):35-9.

35. Ross H, Swinson R, Larkin E, Doumani S. Diagnosing comorbidity in substance abusers. Computer assessment and clinical validation. J Nerv Ment Dis. 1994;182(10):556-63.

36. King C, Van Hasselt V, Segal D, Hersen M. Diagnosis and assessment of substance abuse in older adults: current strategies and issues. Addict Behav. 1994;19(1):41-55.

37. McHugo G, Caspi Y, Kammerer N, Mazelis R, Jackson E, Russell L, et al. The assessment of trauma history in women with co-occurring substance abuse and mental disorders and a history of interpersonal violence. J Behav Health Serv Res. 2005;32(2):113-27.

38. Carroll KM. Methodological issues and problems in the assessment of substance use. Psychol Assess. 1995;7(3):349-58.

39. Santolaria-Fernández F, Gómez-Sirvent J, González-Reimers C, Batista-López J, Jorge-Hernández J, Rodríguez-Moreno F, et al. Nutritional assessment of drug addicts. Drug Alcohol Depend. 1995;38(1):11-8.

40. Westermeyer J. Cultural aspects of substance abuse and alcoholism. Assessment and management. Psychiatr Clin North Am. 1995;18(3):589-605.

41. Moffett L, Steinberg S, Rohde P. Personality assessment of substance-dependent patients in a therapeutic community. J Subst Abuse Treat. 1996;13(2):127-34

42. Vasconcelos T. [Substance dependence. Evaluation of risk and planning of care] Servir. 1996;44(5):247-50.

43. Hiller ML, Knight K, Simpson DD. An assessment of comorbid psychological problems in a residential criminal justice drug treatment program. Psychol Addict Behav. 1996;10(3):181-9

44. Wansi E, Sam-Abbenyi A, Befidi-Mengue R, Enyme F, Ntone F. Rapid assessment of drug abuse in Cameroon. Bull Narc. 1996;48(1-2):79-88.

45. Planeta CdS, Delucia R, Aizenstein ML. On the evaluation of drug dependence in experimental and clinical models. Rev Cienc Farm. 1997;18(2):187-95.

46. Blow F, Barry K, BootsMiller B, Copeland L, McCormick R, Visnic S. Longitudinal assessment of inpatient use and functioning of seriously mentally ill veterans with and without co-occurring substance use disorders. J Psychiatr Res. 1998;32(5):311-9.

47. Addiction. Screening, diagnosis, and assessment. Rep Group Adv Psychiatry. 1998(142):11-33.

48. Badr I, Farghaly A, Koura M, Mohamed H, Hassan E, Kotkat A. Health status assessment of drug addicts in Alexandria. J Egypt Public Health Assoc. 1998;73(34):275-96.

49. Mendelson J, Mello N. Diagnostic evaluation of alcohol and drug abuse problems in women. Psychopharmacol Bull. 1998;34(3):279-81.

50. Souza MAd. Funcionamento psicodinâmico em drogadictos através do Rorschach. Bol Psicol. 1998;48(108):1-12.

51. Evaluation of certain veterinary drug residues in food. Fiftieth report of the joint FAO/WHO Expert Committee on Food Additives. World Health Organ Tech Rep Ser. 1999;888:i-vii, 1-95.

52. Castel S, Formigoni MLOS. Escalas para avaliaçäo de tratamentos de dependência de alcool e outras drogas. Rev Psiquiatr Clin. 1999;26(1 especial):32-7.

53. Cooper D. Substance-use assessment. Prof Nurse. 1999;14(7):449.

54. Mosegui G, Rozenfeld S, Veras R, Vianna C. [Quality assessment of drug use in the elderly]. Rev Saude Publica. 1999;33(5):437-44.

55. Rouse SV, Butcher JN, Miller KB. Assessment of substance abuse in psychotherapy clients: The effectiveness of the MMPI-2 substance abuse scales. Psychol Assess. 1999;11(1):101-7.

56. Silva EAd, Formigoni MLOS. Avaliação do funcionamento familiar em farmacodependências. Rev Psiquiatr Clin. 1999;26(1 especial):38-40.

57. Smit E, Tang A. Nutritional assessment in intravenous drug users with HIV/ AIDS. J Acquir Immune Defic Syndr. 2000;25 Suppl 1:S62-9.

58. Marlowe D, Merikle E, Kirby K, Festinger D, McLellan A. Multidimensional assessment of perceived treatment-entry pressures among substance abusers. Psychol Addict Behav. 2001;15(2):97-108

59. Kan C, Breteler M, van der Ven A, Timmermans M, Zitman F. Assessment of benzodiazepine dependence in alcohol and drug dependent outpatients: a research report. Subst Use Misuse. 2001;36(8):1085-109.

60. Menninger J. Assessment and treatment of alcoholism and substance-related disorders in the elderly. Bull Menninger Clin. 2002;66(2):166-83.

61. Grohman K, Fals-Stewart W. The detection of cognitive impairment among substance-abusing patients: the accuracy of the neuropsychological assessment battery-screening module. Exp Clin Psychopharmacol. 2004;12(3):200-7.

62. Griffiths R, Bigelow G, Ator N. Principles of initial experimental drug abuse liability assessment in humans. Drug Alcohol Depend. 2003;70(3 Suppl):S41-54. 
63. Oliveira ERNd, Marin IC, Ferruzzi L, Tenório MFS, Trindade E. Avaliação dos hábitos alimentares e dos dados antropométricos de dependentes químicos. Arq Cienc Saude Unipar. 2005;9(2):91-6.

64. Cardoso EdS, Pruett SR, Chan F, Tansey TN. Substance abuse assessment and treatment: the current training and practice of APA Division 22 members. Rehabil Psychol. 2006;51(2):175-8.

65. Funada M, Aoo N. [Evaluation of drug dependence: use of a conditioned place preference paradigm]. Nippon Yakurigaku Zasshi. 2007;130(2):128-33.

66. Vanem P, Krog D, Hartmann E. Assessment of substance abusers on the MCMIIII and the Rorschach. Scand J Psychol. 2008;49(1):83-91.

67. Degenhardt L, Bohnert K, Anthony J. Assessment of cocaine and other drug dependence in the general population: "gated" versus "ungated" approaches. Drug Alcohol Depend. 2008;93(3):227-32.

68. Brasiliano S. Fatores psicológicos no abuso e dependência de drogas. In: Andrade AG, Nicastri S, Tongue E, editores. Drogas: atualização em prevenção e tratamento. São Paulo: Lemos; 1993. p. 79-84.

69. Prochaska JO, DiClemente CC. Transtheoretical therapy: toward a more integrative model of change. Psychother Theory Res Pract. 1982;19(3):276-88.

70. Kessler FHP. Psicoterapias para o alcoolismo. In: Cordioli AV, organizador. Psicoterapias: abordagens atuais. Porto Alegre: Artmed; 2008. p. 585-99.

71. Laranjeira R. Como organizar uma história clínica. In: Figlie NB, editor. Aconsethamento em dependência química. São Paulo: Rocca; 2004.

72. de Azevedo R, Botega N, Guimarães L. Crack users, sexual behavior and risk of HIV infection. Rev Bras Psiquiatr. 2007;29(1):26-30.

73. Bastos F, Cunha C, Bertoni N. [Use of psychoactive substances and contraceptive methods by the Brazilian urban population, 2005]. Rev Saude Publica. 2008;42 Suppl 1:118-26.

74. Nappo S, Sanchez Z, Oliveira L, Santos S, Coradete Jr J, Pacca J, et al. Comportamento de risco de mulheres usuárias de crack em relação às DST/AIDS. São Paulo: Centro Brasileiro de Informações sobre Drogas Psicotrópicas; 2004.

75. Nuttbrock L, Rosenblum A, Magura S, Villano C, Wallace J. Linking female sex workers with substance abuse treatment. J Subst Abuse Treat. 2004;27(3):233-9

76. Morawska J, Satora L. [Assessment of the legal awareness regarding drug consumption]. Przegl Lek. 2004;61(4):328-31.

77. da Costa J, de Sousa M, Magalhaes T, Ribeiro C, Grams A, Ramon J, et al. Assessment of drug addiction. The experience of the Medico Legal Institute of Oporto. Med Law. 2000;19(2):309-19.
78. Zilberman M, Blume S. [Domestic violence, alcohol and substance abuse]. Rev Bras Psiquiatr. 2005;27 Suppl 2:S51-5.

79. da Silva Lima A, Fleck M, Pechansky F, de Boni R, Sukop P. Psychometric properties of the World Health Organization quality of life instrument (WHOQoLBREF) in alcoholic males: a pilot study. Qual Life Res. 2005;14(2):473-8.

80. da Silva Lima A, Fleck M. Qualidade de vida e alcoolismo. In: Fleck M, editor. A avaliação da qualidade de vida: guia para profissionais de saúde. Porto Alegre: Artmed; 2008. p. 115-122.

81. Kessler R. The epidemiology of dual diagnosis. Biol Psychiatry. 2004;56(10):730-7

82. Salloum IM. Issues in dual diagnosis [Internet]. MedscapeCME, Accreditation Council for Continuing Medical Education; 2005. Available from: http://cme. medscape.com/viewarticle/507192

83. Lehman A, Myers C, Corty E. Assessment and classification of patients with psychiatric and substance abuse syndromes. 1989. Psychiatr Serv. 2000;51(9):1119-25.

84. Trezza G, Popp S. The substance user at risk of harm to self or others: assessment and treatment issues. J Clin Psychol. 2000;56(9):1193-205.

85. Kubarych TS, Aggen SH, Hettema JM, Kendler KS, Neale MC. Assessment of generalized anxiety disorder diagnostic criteria in the National Comorbidity Survey and Virginia Adult Twin Study of Psychiatric and Substance Use Disorders. Psychol Assess. 2008;20(3):206-16.

86. Grilo C, Becker D, Anez L, McGlashan T. Diagnostic efficiency of DSM-IV criteria for borderline personality disorder: an evaluation in Hispanic men and women with substance use disorders. J Consult Clin Psychol. 2004;72(1):126-31.

87. Bischof G, Rumpf H, Meyer C, Hapke U, John U. Influence of psychiatric comorbidity in alcohol-dependent subjects in a representative population survey on treatment utilization and natural recovery. Addiction. 2005;100(3):405-13.

88. Drake R, ONeal E, Wallach M. A systematic review of psychosocial research on psychosocial interventions for people with co-occurring severe mental and substance use disorders. J Subst Abuse Treat. 2008;34(1):123-38.

89. Flynn P, Brown B. Co-occurring disorders in substance abuse treatment: issues and prospects. J Subst Abuse Treat. 2008;34(1):36-47.

90. Dougherty D, Mathias C, Marsh D, Moeller F, Swann A. Suicidal behaviors and drug abuse: impulsivity and its assessment. Drug Alcohol Depend. 2004;76 Sup$\mathrm{pl}:$ S93-105.

91. Formigoni ML, Castel S. Escalas de avaliação de dependência de drogas: aspectos gerais. Rev Bras Psiquiatr. 1999;26(1)5-31. 